
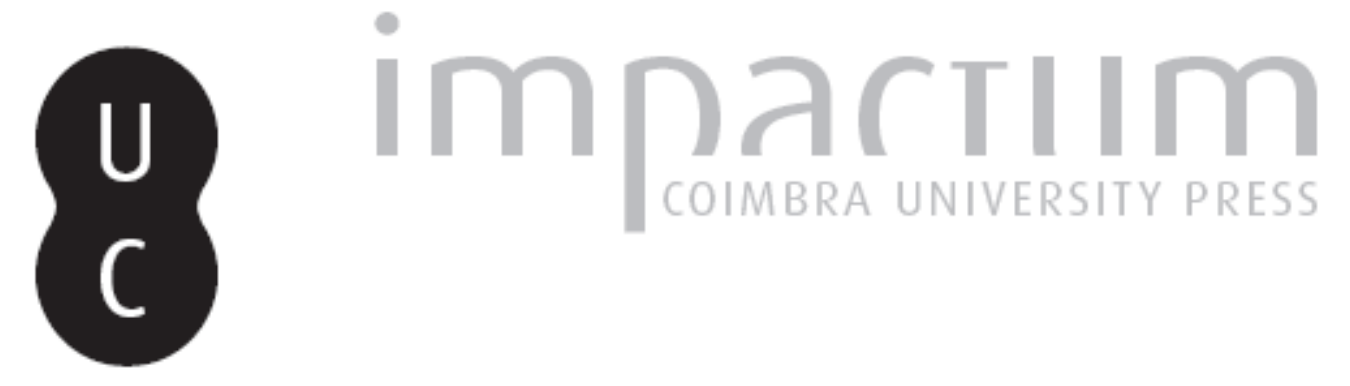

\title{
La dialéctica en Proclo
}

\section{Autor(es): Garay, Jesús de}

Publicado por: Annablume Clássica

URL persistente:

URI:http://hdl.handle.net/10316.2/24480

DOI:

DOI:http://dx.doi.org/10.14195/1984-249X_5_8

Accessed : $\quad$ 26-Apr-2023 13:56:23

A navegação consulta e descarregamento dos títulos inseridos nas Bibliotecas Digitais UC Digitalis, UC Pombalina e UC Impactum, pressupõem a aceitação plena e sem reservas dos Termos e Condições de Uso destas Bibliotecas Digitais, disponíveis em https://digitalis.uc.pt/pt-pt/termos.

Conforme exposto nos referidos Termos e Condições de Uso, o descarregamento de títulos de acesso restrito requer uma licença válida de autorização devendo o utilizador aceder ao(s) documento(s) a partir de um endereço de IP da instituição detentora da supramencionada licença.

Ao utilizador é apenas permitido o descarregamento para uso pessoal, pelo que o emprego do(s) título(s) descarregado(s) para outro fim, designadamente comercial, carece de autorização do respetivo autor ou editor da obra.

Na medida em que todas as obras da UC Digitalis se encontram protegidas pelo Código do Direito de Autor e Direitos Conexos e demais legislação aplicável, toda a cópia, parcial ou total, deste documento, nos casos em que é legalmente admitida, deverá conter ou fazer-se acompanhar por este aviso. 

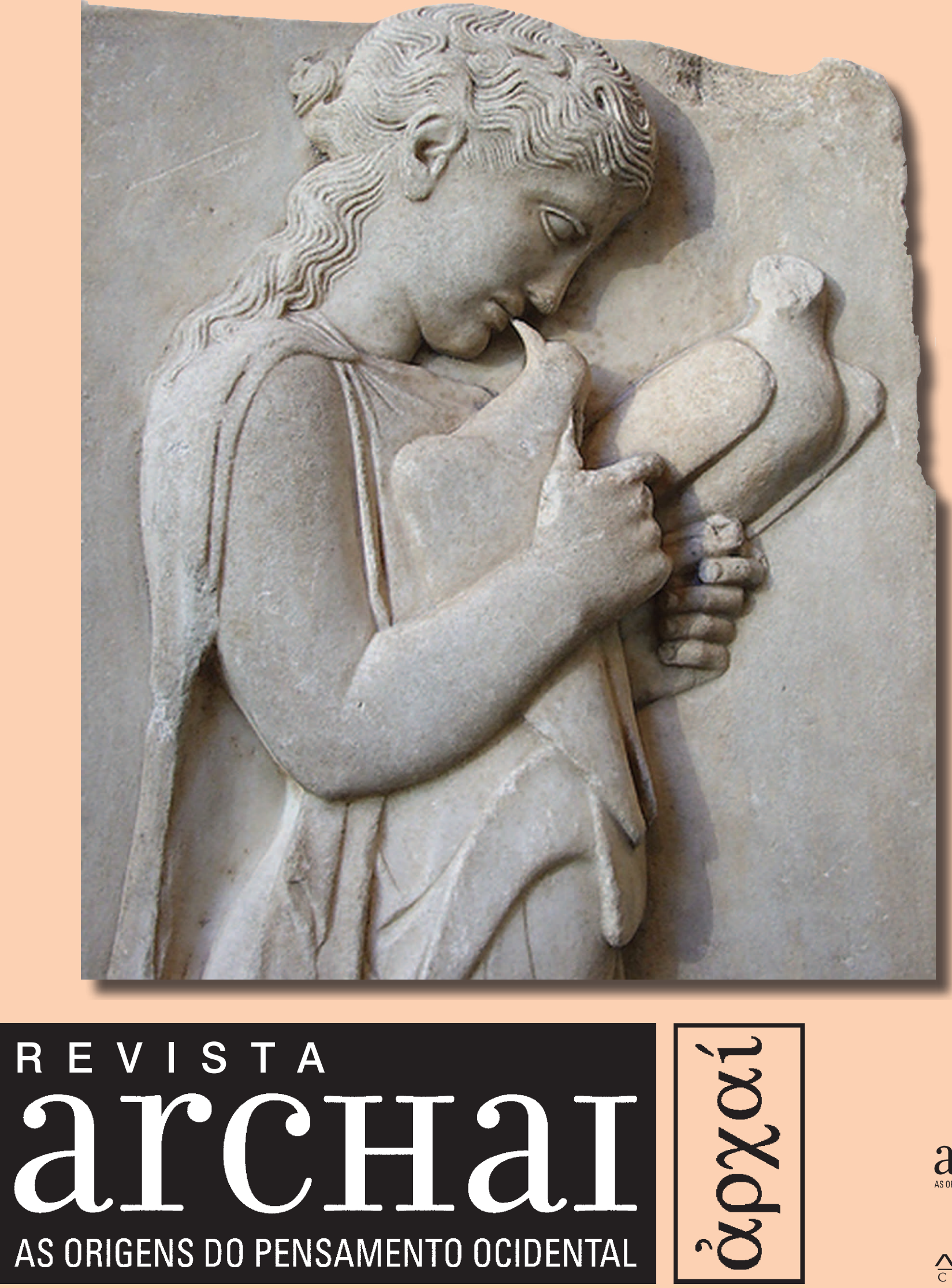


\section{LA DIALÉCTICA EN PROCLO}

RESUMO: Análisis del concepto de dialéctica en Proclo, a partir de los libros II, V y VII del Comentario al Parménides. Se consideran tres aspectos: $1^{\circ}$ ) La dialéctica como método. $2^{\circ}$ ) La dialéctica como perspectiva particular de la totalidad del universo. $3^{\circ}$ ) La dialéctica como orden de las negaciones.

PALABRAS CLAVE: Proclo, dialéctica, negación.

ABSTRACT: Analysis of the concept of dialectics in Proclus, specially in Commentary on Parmenides II, V and VII. It considers three aspects: 1) Dialectics as method. 2) Dialectics as a particular perspective of universe. 3rd) Dialectics as order of negations. KEYWORDS: Proclus, dialectics, negation.

\section{Jesús de Garay ${ }^{1}$}

\section{Introducción}

2. Cf. In Prm., V, 984,27: para Aristóteles la dialéctica sería únicamente un «método argumentativo»

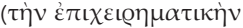
$\mu \varepsilon \dot{\theta}$ oðov). En general, cf. In Prm., V, 984,24-985,11.
$\mathrm{E}_{\text {l concepto de dialéctica tiene una larga }}$ historia no siempre bien conocida. Uno de los pensadores más determinantes en esta historia del concepto de dialéctica es Proclo, neoplatónico ateniense pagano del siglo $\mathrm{V}$ d. JC. Siguiendo la tradición platónica, concibe la dialéctica como el método científico por antonomasia, que permite resolver las oposiciones y superar las perspectivas unilaterales. Me atendré en especial a los libros II, V y VII de su Comentario al Parménides.

\section{La dialéctica como método}

Proclo es fiel a la identificación platónica de la dialéctica como saber necesario, que permite descubrir los principios en su vinculación necesaria de causas y efectos. Y reprocha a Aristóteles y otros filósofos haber minusvalorado el alcance de la dialéctica. El «genial» Aristóteles -frente al «divino» Platón- habría descrito la dialéctica sólo en sus funciones propedéuticas ${ }^{2}$. No obstante, la objeción de Proclo a Aristóteles no le impide integrar dentro de la dialéctica la doctrina de la ciencia expuesta por Aristóteles en los Analíticos. 
Frente a la dialéctica aristotélica subraya su carácter de ciencia y no de opinión probable ${ }^{3}$. Por otra parte, los cuatro métodos en los que se resume la dialéctica son el análisis, demostración, definición y división. Todos ellos se orientan a descubrir los principios primeros de lo que todo deriva, y en ese sentido todos tienen una dimensión de análisis ${ }^{4}$.

El uso de cada uno de estos procedimientos de la dialéctica no está prefijado de antemano ${ }^{5}$. Para algunas cuestiones es útil la división, pero para otras resulta más oportuna la demostración o la definición. En un momento conviene establecer las consecuencias que siguen de unos determinados principios, pero en otro se debe proceder a la inversa determinando las causas a partir de los efectos. En todo caso, lo esencial de la dialéctica, según Proclo, consiste en su carácter necesario, que posibilita establecer conclusiones firmes y no meras opiniones. El objetivo de la dialéctica es siempre el mismo, y remite a la doctrina de la ciencia aristotélica: descubrir las causas de cada cosa ${ }^{6}$.

La dialéctica presenta un doble aspecto. Por un lado expresa el orden de la realidad misma:

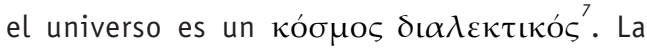
dialéctica no es un discurso meramente lógico distinto de la realidad, sino que dialéctica y realidad se corresponden. Al modo spinozista, se puede decir que ordo et conexio rerum est ordo et conexio idearum. $\mathrm{Y}$ este orden es dialéctico, o lo que es lo mismo según Proclo, es un orden triádico y circular $^{8}$. Cualquier realidad puede ser considerada desde una triple consideración: como permaneciendo idéntica en sí misma ( $\mu$ oví), como desplegando su potencialidad de muchas formas

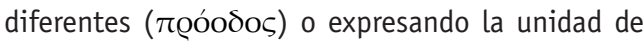

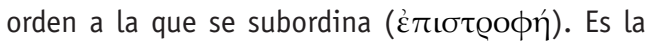
misma realidad pero considerada de tres modos distintos. Pues bien, ese proceder de la realidad -y en especial ese retorno hacia la unidad- ostenta un carácter dialéctico. Es decir, la realidad se despliega siguiendo un orden racional de causas racional-dialéctico. De este modo, la realidad según Proclo siempre puede presentarse como un círculo, es decir, como un proceso ya cumplido -y siempre realizándose- de carácter cíclico: permanecerproceder-volver.
Pero la dialéctica puede ser examinada no sólo como la realidad misma, sino también como el proceder mismo de la reflexión racional en busca de los principios. Es decir, como una fase -la fase esencial- de la razón humana, que aspira a comprender la verdad de los primeros principios. La perspectiva procliana del conocimiento humano se apoya en la conocida división platónica ${ }^{9}$ : la realidad se divide en dos grandes ámbitos, la región de lo sensible ( $\alpha i \sigma \theta \eta \tau \alpha \dot{)}$ y la región de lo inteligible

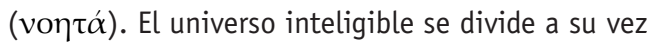
en el propiamente inteligible y en el de lo racional

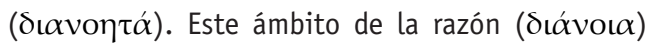
es el característico de la inteligencia humana ${ }^{10} y$ el lugar propio en el que se despliega el proceso dialéctico, es decir, el análisis, la división, la demostración y la definición. En este sentido (como dimensión del pensamiento humano), la dialéctica

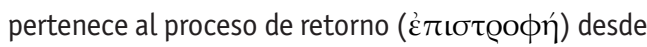
la pluralidad y multiplicidad del mundo físico hacia la unidad del primer principio. Es decir, por medio de la reflexión dialéctica la razón humana es capaz de reproducir el proceso de diferenciación desde la unidad, y en general el orden necesario de todo lo real.

Será entonces, gracias a la razón dialéctica, cuando el pensamiento se hace capaz de la intuición intelectual, es decir, de contemplar directamente la unidad de las formas, sin mediaciones.

Es decir, por encima de la dialéctica y la ciencia, está la inteligencia que capta la unidad sin articulaciones. La metáfora platónica es la visión, concebida como una forma de conocer que conoce de una vez sin recorrer las diferentes partes del objeto visto ${ }^{11}$.

Resulta determinante en el proceso dialéctico el conjunto de las mediaciones ( $\mu \varepsilon \sigma o ́ \tau \eta\rceil, ~ \mu \varepsilon ́ \sigma o \varsigma){ }^{12}$. Ellas son las que establecen los vínculos y la continuidad entre todas las regiones de la realidad. Precisamente uno de los rasgos más destacados de la filosofía de Proclo es su énfasis -ya presente en Jámblico y en Siriano- en la continuidad de lo real, y en la necesidad consiguiente de establecer términos medios entre todos los niveles, de tal modo que no se produzcan saltos ni discontinuidades, tal como Proclo reprocha a Plotino. La reflexión dialéctica
3. In Prm., V, 1003,2-9: Tò

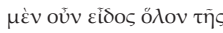
$\delta\llcorner\alpha \lambda \varepsilon \kappa \tau \iota \kappa \eta ̄ \varsigma ~ \mu \varepsilon \boxminus o ́ \delta o v$

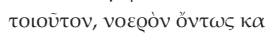

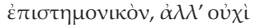

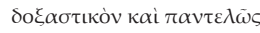

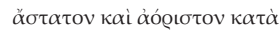

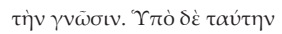

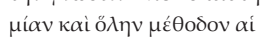

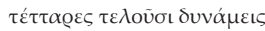

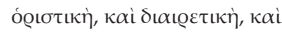

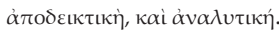
cf. In Euc., 42.

4. In Prm., V, 982,21-28: $\eta \dot{\gamma} \gamma \dot{\alpha} \mathrm{O}$

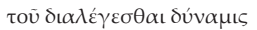

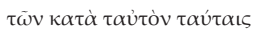

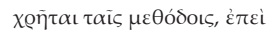

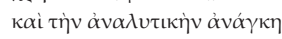

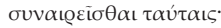

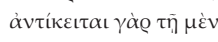
$\dot{\alpha} \pi \circ \delta \varepsilon เ \kappa \tau \iota \kappa \tilde{\eta}, \omega \varsigma \dot{\alpha} \pi \grave{\tau} \tau \tilde{\omega} \nu$ $\alpha i \tau\llcorner\alpha \tau \tilde{\omega} v \dot{\alpha} v \alpha \lambda \hat{v} \operatorname{ov\sigma \alpha } \varepsilon \dot{\varepsilon} \zeta$

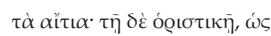

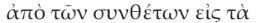

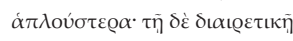

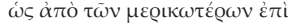
$\tau \grave{\alpha} \kappa \alpha \theta \mathrm{o} \lambda \iota \kappa \omega \dot{\tau} \varepsilon \varrho \alpha$

5. Cf. In Prm., V, 1003,9ss. Cf. In Euc., 18-19.

6. In Prm., V,1006,26-29: $\kappa \alpha i$

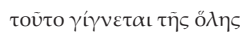

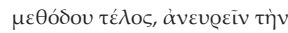

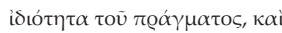

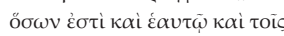

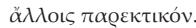

7. Cf. Theol. Plat., I,53,20.

8. Cf. Beierwaltes, W., 1979, passim.

9. Cf. In Euc., 10-11

10. La razón humana no es una Inteligencia divina donde las Formas mismas se manifiestan. La razón humana no es divina sino humana: o lo que es lo mismo, una razón dianoética y dialéctica. Las formas presentes en la inteligencia humana no son las Formas en sí mismas sino «las formas en nosotros», $\tau \dot{\alpha} \dot{\varepsilon} v$

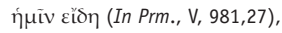
«el lógos en nosotros», ó $\delta \dot{~}$ toũ غ่v ๆ (981,11-12). Las formas que se conocen dialécticamente -esto es, las formas que son definibles- son las formas en el alma y las formas sensibles: $\tau \dot{\alpha} \delta \dot{\varepsilon} \psi v \chi \iota \kappa \grave{\alpha} \kappa \alpha \grave{\imath}$

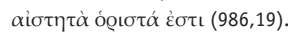
11. Cf. Inst., 170: «Toda inteligencia entiende todas las cosas simultáneamente; pero mientras que la inteligencia noparticipada entiende todo de un modo absoluto, toda inteligencia que está detrás de aquélla conoce todo pero según un solo aspecto»:

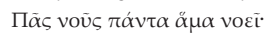

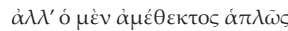

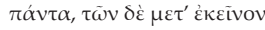

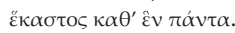


12. Cf. In Prm., V,1024,331025,4; cf. ibid., 1021,5-1023,9 13. Inst.,103. Cf. los comentarios de Dodds $(1962,254)$ : «The general principle of which this is a particular application, viz. that "all things, but in each after its own fashion", is ascribed by Syrianus (in Metaph. 82. I ff) to "the Pythagoreans", and by Iamblichus (ap. Stob. Ecl. I.xlix.31 [866H] to Numenius. Plot. applies it to the relations of intelligibles in general (V.viii.4; II.235.23); it is explicitly laid down by Porphyry (aph.X), and from Iamblichus (cf. Pr., In Tim. I,426,20) onwards is much resorted to. The later school saw in it a convenient means of covering all the gaps left by Plotinus in his derivation of the world of experience, and thus assuring the unity of the sistem: it bridged oppositions without destroying them. Pr. uses it not only to explain the Platonic koinwnía eidwn (in Prm.751 ff.) and to solve Parmenides' difficulties about trascendent Forms (ibid. $928 \mathrm{ff}$.), but also to link together the four material elements (in Tim. II.26.23 ff.); he even adduces it to justify the community of women and children in the Republic (ibid..I.48.24 ff.); and it enables him to evade such a question as "Where does sphericity begin?" by replying that it exists "intellectively" in the demiurge, "intelligibly" in the autodswon, and on still higher planes "secretly" (ibid. II.77: cf.83.161.26,III.285.30, in Prm.812.10). The formula was taken over by ps.-Dion. (e.g. Div.Nom. 4.7) to be echoed at the Renaissance by Bruno, and later given a new significance by Leibniz (cf. Principles of Nature and Grace,3: "Chaque monade est un miroir vivant, représentatif de l'univers suivant son point de vue")».

14. Cf. Inst., 33: «Todo lo que progresa a partir de algo y vuelve a ello tiene una actividad circular.

Pues si vuelve a aquello desde lo que progresa, el fin está unido al principio, y el movimiento es uno y continuo, haciéndose a partir de lo que permanece $y$ volviendo hacia lo que permanece. Por lo tanto todas las cosas proceden circularmente desde las causas hacia las causas»:

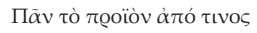

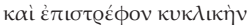

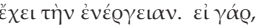
$\dot{\alpha} \phi^{\prime}$ oũ rอó

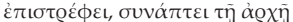

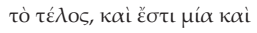

muestra así la continuidad y vinculación de todas las realidades entre sí.

\section{La dialéctica como perspectiva particular de la totalidad del universo}

Esta vinculación universal de toda la realidad se proclama en la proposición 103 de la Stoicheiosis theologiké, que enuncia una de las tesis centrales de Proclo:

«todo está en todo, pero en cada cosa a su manera:

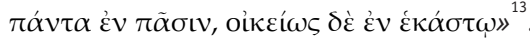

Cada cosa se encuentra vinculada con todas y cada una de las restantes realidades (o procesos) existentes, pero el modo de vinculación del todo con cada cosa es diferente, adecuándose al modo de cada cosa. Por eso mismo, varían las mediaciones que se destacan en cada razonamiento dialéctico, ya que la perspectiva desde la que se despliega el conjunto de lo real es diferente en cada caso.

Cualquier realidad es única porque su relación -sus mediaciones- con todas las demás realidades es una vinculación única y exclusivamente suya. La totalidad de la reflexión dialéctica sobre una realidad resume la singularidad de su esencia ${ }^{14}$. En absoluto resulta anacrónico describir el universo procliano como monadológico ${ }^{15}$. Más aún, en la monadología de Proclo se encuentra posiblemente una de las fuentes indirectas de la monadología leibniziana ${ }^{16}$.

Es decir, la pluralidad de diferencias que se presentan en el universo de Proclo son ante todo diferencias de aspectos o perspectivas ${ }^{17}$. La misma noción de participación ha de ser entendida así: algo es en parte de una manera y en parte de otra ${ }^{18}$. Algo es en un sentido de un modo, y en otro sentido de otro modo. Las diferencias que articula la reflexión dialéctica son diferencias de perspectiva, de puntos de vista. Por eso mismo, algo puede ser una cosa y su contraria, porque en un sentido es una cosa y en otro no. Algo puede ser semejante y desemejante a la vez. 0 igual y desigual. 0 idéntico y diferente. 0 uno y muchos. 0 limitado e infinito. Por eso, se puede aceptar en parte una proposición y refutarla en parte. $\mathrm{Y}$ esto es justamente lo característico de la dialéctica ${ }^{19}$.
Esta es la vía por la que Proclo critica el principio aristotélico de no contradicción. Si Aristóteles afirmaba que es imposible ser y no ser a la vez y en el mismo sentido, Proclo declara que es posible que algo sea y no sea, si es en distinto tiempo y sobre todo si es en distinto sentido ${ }^{20}$. Pero esta diferencia de sentidos ${ }^{21}$ no es algo que caracterice únicamente al discurso racional sino que es propio de la misma realidad. Las relaciones de unas cosas con otras nunca son reversibles. El sentido de cualquier proceso es único e irreductible a cualquier otro. Proclo no renuncia al principio de no contradicción. Muy al contrario, subraya su validez reiteradamente ${ }^{22}$. Pero a la vez, insiste en su insuficiencia para mostrar la realidad en sus diferencias de sentido.

El inicio de la reflexión dialéctica, según Proclo, hay que situarlo en el conocimiento de sí, esto es, en el cumplimiento del mandato délfico: $\gamma \nu \tilde{\omega} \theta$ ı $\sigma \varepsilon \alpha v \tau o ́ v$, conócete a ti mismo. Si todo está en todo, también la totalidad está presente en el alma humana, y es posible descubrirla. $Y$ en particular está presente en lo que Proclo denomina

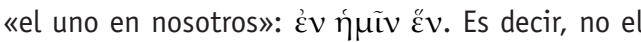
Uno en sí mismo (frente a Plotino), sino el reflejo del Uno en nosotros ${ }^{23}$. Este «uno en nosotros» no es tampoco el «uno del alma», ni el «uno de la inteligencia» sino que es el uno que unifica todas las potencias y realidades de cada individuo singular, incluida su materia y corporalidad. Este «uno en nosotros» vale de punto de partida para el progreso dialéctico, en tanto que ofrece una base de identidad para el progreso del conocimiento, estableciendo diferencias y definiciones de todo el horizonte que se manifiesta ante cada uno de nosotros.

De este modo la visión de la totalidad de lo real que aparece ante el individuo humano es necesariamente un punto de vista sobre la totalidad de lo real. Según Proclo, esto no significa caída en la opinión, sino establecimiento de la verdad necesaria desde una perspectiva singular. Sin duda no es el punto de vista del Uno, pero no por ello se anula el valor de verdad. Cada individuo humano es todo, pero lo es según su modo humano, modo que implica lo intelectual, lo anímico y lo físico material. 
En todo caso, el universo se va ampliando mediante la continuada reflexión a partir del puro

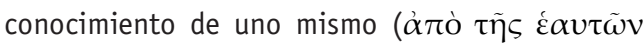
$\kappa \alpha \theta \alpha \varrho \alpha ́ \varsigma \gamma \nu \omega \dot{\sigma} \sigma \omega \omega \varsigma)^{24}$. El ser humano además ofrece un panorama del universo particularmente completo: es de un modo muy específico microcosmos, porque integra dentro de sí todos los niveles de realidad, desde el Uno hasta la Materia. En el planteamiento de Proclo esta doctrina hermética del hombre microcosmos tiene una particular validez ya que cada causa superior tiene un alcance mayor que las causas subordinadas: aquí también se distancia de Plotino, ya que no hay una derivación causal continua desde el Uno hasta la Materia, sino que por ejemplo sólo el Uno es causa de la Materia, del mismo modo que sólo el Ser y el Uno (pero no la Vida ni la Inteligencia) son causa de los seres materiales no vivos, etc.

La singularidad del caso del ser humano es que en él confluyen todos los niveles de realidad, desde el Uno hasta la Materia, y por consiguiente su perspectiva es particularmente completa. Por consiguiente, en el caso del alma humana estamos ante una racionalidad que abarca la totalidad de lo real, según el modo humano: ese modo específicamente humano es la racionalidad dialéctica

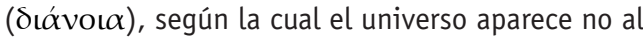
modo de los dioses, ni al modo del ser ni de la vida ni del voũs, sino al modo del alma, y en concreto de un alma individual ligada a un cuerpo mortal.

La dialéctica, por consiguiente, no es simplemente la realidad misma del cosmos, ni tampoco es asimilable sin más a la غ̇ंı todo esto, pero lo al modo humano de la racionalidad

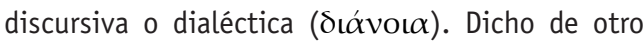
modo, el punto de vista que el hombre tiene del universo es un punto de vista dialéctico, y ahí estriba la especificidad de la perspectiva humana. Todo está en todo, pero en cada realidad lo está según su modo particular: y el modo particular del hombre es la razón discursiva y dialéctica, esto es, como reflexión particular articulada por una multiplicidad de mediaciones.

Ciertamente Proclo no cierra la posibilidad de un progreso para el pensamiento humano que vaya más allá de la razón dianoética, y que alcance la intuición intelectual, el contacto con los dioses e incluso una cierta unión con el Uno. Pero esas posibilidades dependen ya de particulares revelaciones divinas o de acciones teúrgicas. Más aún en último término el Uno en sí mismo es incognoscible e inexpresable, tanto que es preciso negarle incluso la denominación de Uno, de Bien, y por supuesto de Ser. El deseo de unificación presente en la razón humana no es suficiente para que sea satisfecho, porque siempre queda anclado en ese «uno en nosotros», que determina el modo humano de comprender.

\section{La dialéctica como orden de las negaciones}

El único modo apropiado de pensar el Uno es la negación ${ }^{25}$. Y en esa misma medida, la negación se convierte en el modo específicamente humano (racional, discursivo, dialéctico) de pensar ${ }^{26}$. Puesto que si el deseo del Uno es el que dirige y orienta todo pensamiento, y si la negación es el modo apropiado de pensar el Uno, entonces todo pensar humano está regulado por la negación. Más aún, toda afirmación procede de la negación. El progreso en el conocimiento se establece mediante un orden determinado de negaciones. La negación es productiva ${ }^{27}$ : al decir lo que algo no es, abrimos un espacio para afirmar posibles predicados.

El progreso racional sigue el orden de series antitéticas, de acuerdo con la doctrina de la participación. Cuando se afirma que algo en parte no es y en parte sí lo es, se está estableciendo la negación de ambos lados de la oposición: ni es ni no es. Pero eso exige a la razón avanzar hacia el nivel superior de lo participado (y más allá al nivel de lo imparticipable). 0 dicho de otro modo, también típicamente procliano: algo en un sentido es y en otro no es, y por tanto hay que negar igualmente que es y que no es, para establecer a continuación desde dónde se ha establecido esa diferencia de sentidos opuestos.

Este es expresamente el planteamiento del Comentario al Parménides de Proclo: establecer el orden de las negaciones de la primera hipótesis («que el Uno no es»). Y a partir de dicho orden de negaciones, poder -de un modo secundario- formular

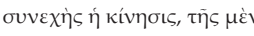

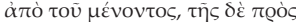

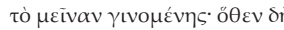

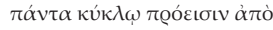

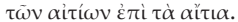

Cada proceso circular es único y revela un aspecto distinto de la realidad precisamente de este modo dinámico-circular y no de un modo estático. El aspecto único y particular de cada cosa es el proceso mismo. La diferencia que singulariza cada cosa es el sentido de su proceso cíclico. La singularidad de una cosa es su diferencia de sentido: esto es, la diferencia del sentido de su proceso circular. Cf. Trouillard 1972, 20: «Ainsi chaque point de l'univers intelligible est d'une manière originale le tout, non seulement parce qu'il est un foyer original de relations, mais surtout parce qu'il effectue en lui-même le processus constituant du tout». 15. Cf. Trouillard 1955, 311: «Mais chaque moi est intrinsèquement differencié. Il appartient à un ordre défini (dieux, esprits, âmes), et il fait éclore ce dernier sous un mode unique. La singularisation de la monade exige que, présents en chacune, tous les degrés composent ensemble à chacune sa loi propre et sa situation intelligible. Tous doivent alors conserver sous ces variations leurs proportions mutuelles. C'est la seule manière pour eux d'être à la fois mêmes et autres».

16. Una de las vías de la recepción de Proclo en Leibniz es Angelo Steuco (Cf. Ch. B. Schmitt, 1996), quien apoyándose en Proclo, escribe su Philosophia perennis, que influye a su vez en Leibniz. El mismo Leibniz menciona en algunas cartas sus lecturas directas de Proclo, (por ejemplo, a Fabricius, a Johann Bernoulli o a La (roze), o también en Prèceptes pour avancer les sciences (1680), destacando el valor del análisis de los axiomas o primeros principios que lleva a cabo Proclo. Acerca de las fuentes de Leibniz, cf. B. Orio de Miguel (2002 y 2004).

17. En relación a In Prm. 755,514, comenta Dillon $(1987,97)$ : «It is best, perhaps, to see the Platonic Forms as distinct "points of view" within an integrated system, each containing the whole, but from a unique perspective». A la vez las diversas formas de comunión entre las Formas están simbolizadas por los matrimonios entre los dioses (Dillon 1987, 99). 
18. Cf. Inst., 2: «Todo lo que participa del Uno, es uno y no es

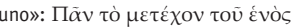

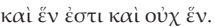
19. Cf. In Prm., V, 989,20-29. 20. Cf. In Prm., II, 726,41-727,2: «Es imposible que las mismas cosas sean a la vez semejantes y desemejantes en el mismo

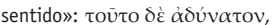

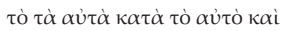
ő No obstante, poco después $(727,35$ y 38$)$, señala que no hay nada extraño en admitir que algo es a la vez semejante y desemejante, siempre que sea en sentidos distintos ( $\kappa \alpha \tau^{\prime} \alpha \check{\alpha} \lambda \lambda \mathrm{o}$

$\kappa \alpha i ̀ ~ \alpha ̋ \lambda$ o). En general a lo largo del libro II hay frecuentes referencias a cómo pueden existir simultáneamente los contrarios, siempre que se entiendan en diferentes sentidos.

21. Cf. el uso constante que hace Proclo de las diferencias de sentidos en la Institutio theologica. Por ejemplo, respecto a la participación de la unidad,

cf. Inst., 4: algo participa de la unidad, en tanto que (ñ) está unificado. En relación al productor ( $\tau$ ò $\pi \alpha \varrho \alpha ́ \alpha \gamma$ ) y a lo producido

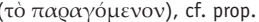
28: en un sentido ( $\pi \tilde{\eta} \mu \dot{\varepsilon} v i ́)$ el producto se distingue del productor y en otro sentido $(\pi \tilde{n}$ $\delta \grave{\varepsilon})$ se identifican. Cf. lo mismo en la prop. 30: $\eta^{\dagger} \mu \dot{\varepsilon} v \ldots . . \eta \eta^{\dagger} \delta \dot{\varepsilon}$. $Y$ la misma diferencia de sentidos se encuentra entre ser según la

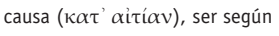
la existencia ( $\kappa \alpha \theta^{\prime}$ v้ $\pi \alpha \varrho \xi\llcorner$ ), y ser según participación ( $\kappa \alpha \tau \dot{\alpha}$ $\mu \varepsilon \theta_{\varepsilon} \varepsilon \xi(v) ;$ cf. esto mismo también en las prop. 65, 118 ó 140. En resumen, la diferencia de sentido se presenta como la noción central que explica en último término otras nociones, como las de causa

o participación. Si todo está

encadenado según este proceso causal, entonces todo está ligado a todo, y todo está de algún modo presente en todo, pero lo está de un modo diferente en cada caso. 22. Cf. In Prm., II, 721,4-729,19. En particular, 726,12-16.

23. Cf. In Prm., VII, 54K 3-19: «Illud attamen querendum quomodo vocamus ipsum, non ens totaliter nominabile,

'unum.' Aut non illud vocamus

sic nominantes, sed eam que in nobis intelligentiam unius. Omnia enim entia primissimam causam

desiderant et habent quandam secundum naturam $\omega \delta i v \alpha$ circa ipsam, et intellectualia et animealia et animata et inanimata et ipsa cum hiis materia. Penes las afirmaciones correspondientes, ya dentro del terreno del Uno-que-es, del Uno-Ser parmenídeo. Pero lo esencial del progreso racional es establecer una deducción a priori desde lo que el Uno no es, pasando por toda la serie de negaciones posibles además del no-ser.

La negación deja abierto un amplio margen para que la razón proyecte ${ }^{28}$ construcciones teóricas que den razón del mundo. Pero la crítica, la negación de una proposición hasta entonces creíble deja paso a la proposición opuesta, que cuando también es negada permite ascender al origen de esa distinción. Si la reóodos es diferenciación,

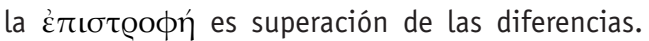
Pero tanto la diferenciación propia del processus como la unificación de las diferencias propia del regressus aluden a diferencias de sentido: en este sentido sí es verdad, pero en este sentido no. Y así sucesivamente, mediante el análisis de los diferentes sentidos o consideraciones de cualquier realidad. Las negaciones representan el carácter productivo y progresivo de las diferencias de sentido. Merced a la negación, al reproche, a la objeción y a la crítica, se puede superar la unilateralidad de una determinada perspectiva. Desde este punto de vista esto es verdad, pero desde ese otro punto de vista no lo es. Las diferencias aludidas por la negación no son negaciones absolutas sino diferencias de sentido, diferencias de aspecto, diferencias de punto de vista o perspectiva.

De ahí el interés de Proclo y en general de la Escuela de Atenas de buscar el acuerdo entre las diferentes tradiciones teológicas y filosóficas ${ }^{29}$. No por un superficial ánimo de eclecticismo sino por un serio empeño crítico de negar universalidad a las posiciones unilaterales.

La dialéctica es circular, como todo el universo procliano. Se parte del conocimiento de sí en busca del reconocimiento del «uno que está en nosotros». Pero ese proceso de reconocimiento del «yo pienso» (oĩ $\mu \mathrm{L}$ ) es un continuo camino de ida y vuelta, yendo en busca de todo lo que no es uno mismo y regresando con esas nuevas articulaciones que de nuevo se asientan en el yo. La circularidad dialéctica no es sino un renovado enriquecimiento de las articulaciones del yo con la totalidad de los otros seres que aparecen ante nosotros. Lo que no hay -frente a la tradición cartesiana modernaes el anclaje en la subjetividad del yo. Porque precisamente el Uno en nosotros va más allá del yo. Los límites del yo son inabarcables precisamente porque el uno es más amplio que el yo. La razón no tiene como sujeto al yo sino al uno: se piensa desde el uno, no desde el yo. Esta al menos sería la objeción principal de Proclo frente a la dialéctica moderna. Más aún, ese «uno» que regula y orienta la discursividad racional no es un dios ni el primero de los dioses (que permanece siempre oculto) sino el uno en nosotros, una unidad a priori que dirige el desarrollo dialéctico.

La razón dialéctica, ascendiendo de mediación en mediación, supera y unifica la unilateralidad de las perspectivas particulares. La negación ejerce de este modo un papel igualmente productivo. De negación en negación, podemos ascender hasta la máxima unidad posible. Las negaciones son previas a cualquier afirmación. La vía racional es primero la crítica de la unilateralidad, y después la propuesta de modelos que se afirman hipotéticamente. Proclo nunca abandona el carácter esencialmente hipotético de la racionalidad dialéctica establecido por Platón ${ }^{30}$.

Todas las perspectivas aparentemente opuestas quedan unificadas sin destruir la pluralidad de los puntos de vista, que permanecen diferenciados y opuestos entre sí. La unidad primera no destruye las diferencias. La unidad de la razón (esto es, el Uno en sí) queda caracterizada así como negación de negación, negatio negationis ${ }^{31}$. El unum in nobis sería la unidad regulativa de la razón, que, mediante la negación de la negación, asciende hacia el non aliud, hacia lo no-otro, la negación de la alteridad, que es uno de los nombres más apropiados para designar al primer principio, tal como propone Nicolás de Cusa siguiendo explícitamente a Proclo.

La razón dialéctica recorre incesantemente la vía de las negaciones, a partir del uno a priori que se encuentra en cada uno de nosotros. Y asciende así de negación en negación hasta intentar alcanzar el objetivo último: la negación de toda negación. Si fuese posible detenernos, sin hipótesis de ninguna clase, en una negación 
última, en una crítica definitiva, la razón dialéctica dejaría paso a la intuición intelectual del Uno en $s i^{32}$, que comprendería todas las perspectivas simultáneamente. $Y$ en tanto que eso no es posible, debemos limitarnos a unificaciones parciales de diferentes perspectivas, aspirando, eso sí, ampliar continuamente el horizonte de la conciencia.

\section{Bibliografía:}

Beierwaltes, W., 1979: Proklos. Grundzüge seiner Metaphysik, Frankfurt a. M.

Breton, S., 1987: «Négation et négativité proclusiennes dans l'oeuvre de Jean Trouillard», en G. Bos-G. Seel (eds.), Proclus et son influence, 81-100.

Cousin, V., ${ }^{2}$ 1864: Procli philosophi Platonici opera inedita, Paris (1820-1827). Reimpr. Hildesheim, 2002.

Dillon, J. M.-Morrow, G. R., 1987: Proclus' Commentary on Plato's Parmenides (in Prm.), Princeton.

Dodds, E. R., '1962: Proclus. The Elements of Theology (Inst.), Oxford (1933).

Friedlein, G., 1873: Procli Diadochi in primum Euclidis elementorum librum commentarii (in Euc.), Leipzig. Reimpr. Hildesheim, 1967.

Klibansky, R.-Labowsky, C., 1953: Parmenides usque ad finem primae hypothesis nec non Procli Commentarium in
Parmenidem, pars ultima adhuc inedita interprete $G$. de Moerbeka (in Prm.), London. (K)

Morrow, G. R., 1970: A Commentary on the First Book of Euclid's Elements (in Euc.), Princeton.

Orio de Miguel, B., 2002: Leibniz y el pensamiento hermético. A propósito de los «Cogitata in Genesim» de F.M. van Helmont, Valencia.

Orio de Miguel, B., 2004: La filosofía de Lady Anne Conway, un proto-Leibniz. "Principia Philosophiae Antiquissimae et Recentissimae», Valencia.

Saffrey, H. D.-Westerink, L. G., 1968-1997: Proclus: Théologie platonicienne, Paris.

Saffrey, H. D., 1992: «Acorder entre elles les traditions théologiques: Une caractéristique du néoplatonisme athénien», E. P. Bos-P. A. Meijier (eds.), On Proclus and his Influence in Medieval Philosophy, Leiden, 35-50.

Schmitt, Ch. B., 1996: «Perrenial Philosophy: From Agostino Steuco to Leibniz», JHI, 27/4,505-532.

Segonds, A.-P., 1985-1986: Proclus: Sur le premier Alcibiade de Platon (in Alc.), Paris.

Steel, C., 1982-1985: Proclus: commentaire sur le Parménide de Platon, Traduction de Guillaume de Moerbeke, Leuven.

Trouillard, J., 1972: L'Un et l'âme selon Proclos, Paris.

Trouillard, J., 1955: «La Monadologie de Proclus», RPL 309-320. quod et illud ostendimus, quod non est per cognitionem eligere le unum. Non enim utique appeteret ipsum et quod cognitionis expers; omnia autem habent per se naturalem circa unum ' $\omega \delta \bar{i} v \alpha$, et etiam anima. Quid enim aliud est le unum quod in nobis quam $\omega \delta$ ivo ${ }^{\prime}$ huius operatio et adiectio? Hanc igitur intrinsecam unius intelligentiam, provolem entem eius quod in nobis unius et velut expressionem, sic nominamus unum. Non illud igitur nominabile, sed quod in nobis unum. Per hoc autem ut convenientissimum ipsi primo circa illud dicimus et insinuamus vicinis. Et enim duplici ente in nobis operatione, hac quidem appetitiva, hac autem inspectiva, et hac quidem etiam hiis que post nos ente innata, hac autem hiis que attendere solum possent sui ipsorum appetitibus, oportebatutique manentem communem omnium neque nostram animam deficere». Asi pues, el Uno en sí es innombrable e incognoscible. Y cuando nos referimos al Uno lo hacemos desde el unum in nobis, esto es desde la intelección del Uno que es propia del alma humana. El unum in nobis al que aludimos cuando nombramos al "uno" no es nuestro concepto del Uno sino el uno deseado por el alma humana, del mismo modo que todos los demás seres que también desean la unidad. No el Uno pensado sino el Uno deseado. Por eso es posible aumentar nuestro conocimiento del Uno, en tanto que reflexionamos sobre la unidad a la que aspiramos, de la que vamos siendo conscientes paulatinamente. En todo caso, el horizonte de nuestro conocimiento queda limitado por la progresiva clarificación en conceptos del deseo humano de unidad y no tanto del Uno en sí mismo.

24. In Alc., 11, l-3

25. Acerca de la negación, cf. especialmente la última parte del libro VII del In Prm., sólo existente en la traducción latina de Moerbecke (cf. Klibansky 1953). 26. Cf. In Prm., VII, 70K 5-12: «Que itaque unius abnegationes, non sunt circa unum. Nichil enim totaliter illi adest, neque ut species, neque ut privatio; sed sicut dicebamus quod nomen hoc, scilicet 'unum,' est eius qui in nobis conceptus, sed non ipsius unius: sic utique dicimus, quod et abnegatio circa hunc est, circa illud autem unum nulla est dictarum abnegativarum conclusionum; sed exaltatum est propter simplicitatem ab omni 
oppositione et omni negatione. Merito ergo in fine apposuit quod abnegationes he non sunt circa unum». Es decir, todas nuestras referencias al Uno se expresan por medio de negaciones, pero propiamente no dicen lo que el Uno mismo es, sino quedan circunscritas al horizonte del unum in nobis, esto es, al deseo del Uno presente en el alma humana. El Uno en sí mismo es ajeno a la negación y por tanto cualquier proposición negativa no expresa nada acerca del Uno. En rigor habría que decir que la dialéctica no es una vía hacia el Uno en sí mismo considerado sino únicamente una vía hacia la unidad presente en nosotros y objeto de nuestros deseos.

27. Cf. In Prm., VII, 72K 23-30: «dicimus quod abnegationes in uno assumens ut generativas affirmationum, sicut dictum est sepe, ut non virtutem putans habere le unum generativam entium omnium -et existentiam substitutivam auferens ab ipso et essentiam- lateas. [...] Ultima negatio uni apposita fert et abnegationes generativas ab ipso. Et hoc est le non possibile esse hec circa unum: le neque potentiam generativam totorum ipsum habere, qualem esse abnegationem dicebamus, etsi igitur generare dicatur». La potencia generativa no pertenece propiamente al Uno porque nada (tampoco el poder de generar) se puede afirmar del Uno, que carece de esencia y de existencia: ese poder de generar todas las cosas pertenece en cambio a la negación, mediante la cual se producen todas las afirmaciones. Cf. Breton, S., 1987, 83-94.

28. La función constructiva de modelos geométricos corresponde propiamente a la imaginación. Acerca de las

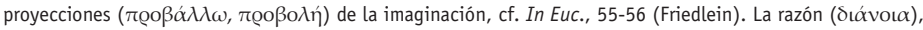
más bien, se sirve de los conceptos utilizados en las negaciones para reconvertirlos en conceptos afirmativos. Por ejemplo, si se ha negado que el Uno es, entonces es que hemos pensado (negativamente) el Uno desde el ser, y por lo tanto ya disponíamos del concepto de ser para negar el Uno; y por lo tanto, podemos afirmar (pensar afirmativamente) el ser en un nivel inferior al del Uno. $Y$ así sucesivamente podemos reconocer una serie de conceptos afirmativos, en tanto que hemos sido capaces de formular una serie de negaciones acerca del Uno. Pero en todo caso, la negación es siempre más universal que la afirmación, por lo que los conceptos afirmativos siempre mantienen un cierto carácter hipotético, puesto que su reconocimiento se basa en la incognoscibilidad (en la negación) del Uno.

29. Cf. Saffrey, H. D., 1992.

30. Cf. Beierwaltes, W., 1979, 253-274.

31. Cf. In Prm., VII, 70-74K. El último paso de la dialéctica es la negación de todas las negaciones previas, es decir, negar que la negación tenga validez para decir algo del Uno. Negar del Uno incluso las mismas negaciones. Este sería el objetivo final de Platón, terminar negando la misma negación. «Merito ergo ultimo et ipsas abnegationes removit ab uno, impossibile dicens has esse circa unum indicibile et incognoscibile existens. Et non mireris, si ubique honorans axiomata contradictionis Plato hic simul mentiri dicit et affirmationes et negationes in uno. [...] Et hoc est quo a Platone differt demonius Aristoteles (72K 1-11). El principio de no contradicción queda así superado en este ámbito en el que son falsas tanto las afirmaciones como las negaciones simultáneas sobre lo mismo: «contradictionem in indicibili quidem simul falsam esse dicendum» (72K 20-21).

32. Cf. In Prm., VII, 74K 14-29: «Propter omnia itaque hec videtur michi ultimo et abnegationes auferre ab uno. Ad ea quidem enim que velut preianualia unius deducet utique nos que per abnegationes hec tota dialectica methodus, auferens omnia inferiora et per ablationem solvens impedimenta speculationis illius, si possibile dicere. Post pertransitum autem per omnia seponere oportet et hanc tamquam valde negotiosam et coattrahentem abnegatorum conceptum, cum quibus non est illi adiacere. Neque enim intellectum est sincere videre preiacentem intelligentiam eorum que post ipsum, neque animam circumdistractam a superinstantia eorum que post animam, neque totaliter cum superinstantia videre aliquid perfecte. Nam superinstantie sunt difficultatem patientis speculationis, propter quod et natura anepistatos, id est sine superinstantia, facit que facit, et scientia dicit que dicit. Tunc autem superinstat, quando dubitat solum et deficit secundum quod est scientia. Sicut igitur in hiis oportet operationem purgari a superinstantia, quamvis per superinstantias perfectam, secundum hec utique et in hiis oportet purgari ab omni dialectica operatione». La reflexión dialéctica debe ser abandonada para pensar el uno, aunque sea necesario previamente recorrerla. Negar la negación significa precisamente abandonar el método dialéctico, sustituir la mediación de la reflexión por la inmediatez de la intuición. Esa inmediatez es precisamente el silencio: «nam per negari et ipse removit omnes abnegationes. Silentio autem conclusit eam que de ipso theoriam» (76K 6-7). Con todo, el silencio no es propiamente una meta sino más bien el límite del pensamiento humano. 